\title{
Research Data Preservation
}

Brett Currier, Bommae Kim, Cynthia Edwards, Courtney Butler

NDSA: DigiPres

October 26, 2017 
Brett D. Currier

Digital Scholarship Librarian \& Library Manager

Center for the Advancement of Data Research in Economics

Federal Reserve Bank of Kansas City

The views expressed herein are solely those of the author and do not necessarily reflect the views of the Federal Reserve Bank of Kansas City or the Federal Reserve System. 


\section{Objections to Content Recruitment}

- Legal Objections

- Copyright

- Contract

- IRB

- Suitability of Compiled Data and Code for release 
Why create
recommendations? 
Looking for De facto Recommendations 


\section{Subset}

- Top 250 journals from combined select Web of Science categories (250 total)

- Top 20 journals from individual select Google Scholar Metrics categories (271 total after de-duplication)

- Top 250 journals from IDEAS/RePEc (250 total)

- All journals with at least one FRBKC interaction (publication or service) (152 total)

- 923 journal titles in subset 


\section{Select Categories}

\section{Google Scholar Metrics}

- Accounting \& Taxation

- Business, Economics \& Management (general)

- Development Economics

- Economic History

- Economic Policy

- Economics

- Entrepreneurship \& Innovation

- Finance

- Game Theory \& Decision Science

- Human Resources \& Organizations

- International Business

- Marketing

- Strategic Management

- Tourism \& Hospitality

- Probability \& Statistics with Applications

\section{Web of Science}

- Agricultural Economics \& Policy

- Business

- Business, Finance

- Economics

- Industrial Relations \& Labor

- Management

- $\quad$ Statistics \& Probability 


\section{Additional Selection}

- Cross-referenced all four sources, included any journal that appeared more than once (220 journals)

- Added remaining journals in top 50 from Google Scholar Metrics, IDEAS/RePEc, and Web of Science (20 journals)

- Added remaining journals with 2+ FRBKC interactions (5 journals)

- 245 journals before exclusions 


\section{Exclusions}

- 9 exclusions

- Journals that were not peer-reviewed

- Journals that did not accept articles relating to data/economics and related disciplines

- Journals no longer in print

- Federal Reserve publications

- 236 journals in final sample 


\section{Text Analysis}

- Identified and downloaded journal policies, author instructions or guidelines, and style guides from journal website(s)

- Analyzed text to identify inclusion in 2 categories:

- Data Availability or Replication Policy

- Supplemental File Recommendations or Requirements 


\section{Supplemental File \\ Recommendations or Requirements*}

Recommendations or requirements, including:

- Restrictions on file format, size, or specifications

- File Naming Conventions

- Defining data or code for the supplemental file

Included even if these features were entirely optional 


\section{Results}

- 80 journals (34\%) have a data availability or replication policy

- 190 journals (81\%) have some recommendation or requirement for supplemental materials 


\section{Archival Standards}

- 10 year half life

- 181 journals (77\%) have guidelines consistent with preservation standards

- Cross referencing against:

- Library of Congress

- National Archives

- Smithsonian 
Preliminary

Recommendations 


\section{Computer Code}

\section{Category}

Format

Which code?

\section{Copyright Notices}

Licensing Terms

\section{Recommendation}

\section{Still determining}

Programs used to run the final models

Intermediate program files

Computer program or code used to convert the raw data into the final dataset used in the analysis plus a brief description that enables other researchers to use this program

See the Fed License

See the Fed License 


\section{Computer Code}

\section{Category}

Author Contributions Checklist

\section{Recommendation}

\section{Abstract}

How Delivered (R package, Shiny, etc.)

License information

Link to code

\section{Version Information}

Hardware Requirements

Supporting Software requirements 


\section{Data}

\section{Category}

Data definition

File Format

Public (but Proprietary) Databases

Data File Layout

\section{Copyright Notices}

Licensing Terms

File Size

\section{Recommendation}

Data sets used to run the final models

Intermediate data files (Optional)

Raw data files (Reproducible)

CSV

A precise description of the databases from which the data was drawn

How the propriety data can be obtained by others

Variables and commands should be clearly labeled

See the Fed License

See the Fed License

20 Gigs 


\section{Experimental Papers}

\section{Additional Documents}

Sample images of any advertising stimuli used;

Screen-capture of any computer interface used;

Pertinent details about the procedure (e.g., instructions, filler task);

Additional details about the method, analyses and results as indicated in the appendix to this document

Flowchart of the data collection instrument

Interviewing Guide

Coding Instruments

The subject pool and recruiting procedures.

The experimental technology - when and where the experiments were conducted; by computer or manually;

Any procedures to test for comprehension before running the experiment, including the use of practice trials and quizzes

\section{Additional Documents}

Provide the instrument

Results of pilot tests

Results of earlier experiments

Describe Experimental instruments

Describe experimental collection procedures if data is collected by a third party

Original instructions

Information regarding subject eligibility or selection

Methodological Details Appendix

Full phrasing of the questions and scales used for the reported findings;

Full text of any scenarios or vignettes used; 


\section{Experimental Papers}

\section{Additional Documents}

Detailed statement of protocols

Samples of permission forms and record sheets

Copies of instructions and slides and/or transparencies used to present instructions

Source code for computer programs used to conduct the experiment and to analyze the data

Screen shots showing how the programs are used

The experimental data together with adequate documentation of the format

\section{Additional Documents}

Matching procedures, especially for game theory experiments

Subject payments, including whether artificial currency was used, the exchange rate, show-up fees, average earnings, lotteries and/or grades

The number of subjects used in each session and, where relevant, their experience

Timing, such as how long a typical session lasted, and how much of that time was instructional

Any use of deception and/or any instructional inaccuracies 


\section{Figures, Images, Tables, Screenshots}

\section{Category}

File Names

Resolution

File Types

Color Illustrations

Black and White illustrations

Copyright Notices

Licensing Terms

\section{Recommendation}

Should include the Figure Name and number according to their sequence in the text

$300-1200$ DPI

Publication standard. No official position

GIF, JPEG, PDF, PDFA, PNG, and TIF

The dominant 2 formats are CYMK and RGB and different publications have different standards. Those two standards are CYMK and RGB.

Publication standard. No official position Not RGB color tones

See the Fed License

See the Fed License 


\section{Read Me}

\begin{tabular}{|l|l|}
\hline Category & Recommendation \\
\hline Author Checklist & Abstract \\
\hline & Availability \\
& Description \\
& Permissions \\
& Licensing Information \\
& Link to data \\
& Data Provenance \\
& File Format \\
& Version Information \\
& Unique Identifier (DOI) \\
&
\end{tabular}




\section{Read Me}

\begin{tabular}{l|l}
\hline Category & Recommendation \\
\hline Manifest & A list of all files in the supplement \\
& A list of proprietary software required for the file (if relevant) \\
& Brief description \\
\hline File Format & PDF or PDFA \\
\hline Timing & 5 to 6 years \\
\hline
\end{tabular}




\section{Next Steps}

- Code Annotation

- Data Annotation 

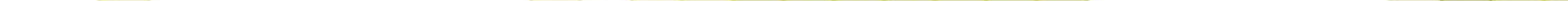